\title{
Landscape relatedness: detecting contemporary fine-scale spatial structure in wild populations
}

\author{
Anita J. Norman 1 - Astrid V. Stronen • Geir-Arne Fuglstad • \\ Aritz Ruiz-Gonzalez • Jonas Kindberg • Nathaniel R. Street • \\ Göran Spong
}

Received: 16 December 2015/Accepted: 5 August 2016/Published online: 18 August 2016

(C) The Author(s) 2016. This article is published with open access at Springerlink.com

\begin{abstract}
Context Methods for detecting contemporary, finescale population genetic structure in continuous populations are scarce. Yet such methods are vital for ecological and conservation studies, particularly under a changing landscape.

Objectives Here we present a novel, spatially explicit method that we call landscape relatedness (LandRel). With this method, we aim to detect contemporary, fine-scale population structure that is
\end{abstract}

\section{A. J. Norman $(\varangle) \cdot$ J. Kindberg $\cdot$ G. Spong}

Department of Fish, Wildlife and Environmental Studies, Swedish University of Agricultural Sciences, Umeå,

Sweden

e-mail: anitajnorman@gmail.com

\section{A. J. Norman}

Department of Life Sciences, San Diego Zoo Global, San Diego, USA

\section{A. V. Stronen}

Section of Biology and Environmental Science,

Department of Chemistry and Bioscience, Aalborg

University, Aalborg, Denmark

\section{G.-A. Fuglstad}

Department of Mathematical Sciences, Norwegian University of Science and Technology, Trondheim, Norway

\section{A. Ruiz-Gonzalez}

Department of Zoology and Animal Cell Biology,

University of the Basque Country, UPV/EHU,

Vitoria-Gasteiz, Spain sensitive to spatial and temporal changes in the landscape.

Methods We interpolate spatially determined relatedness values based on SNP genotypes across the landscape. Interpolations are calculated using the Bayesian inference approach integrated nested Laplace approximation. We empirically tested this method on a continuous population of brown bears (Ursus arctos) spanning two counties in Sweden.
A. Ruiz-Gonzalez
Systematics, Biogeography and Population Dynamics Research Group, Lascaray Research Center, University of the Basque Country, UPV/EHU, Vitoria-Gasteiz, Spain
J. Kindberg
Norwegian Institute for Nature Research, Trondheim, Norway
N. R. Street
Umeå Plant Science Centre, Department of Plant
Physiology, Umeå University, Umeå, Sweden
G. Spong
Fisheries, Wildlife and Conservation Biology Program, Department of Forestry and Environmental Resources, North Carolina State University, Raleigh, NC, USA


Results Two areas were identified as differentiated from the remaining population. Further analysis suggests that inbreeding has occurred in at least one of these areas.

Conclusions LandRel enabled us to identify previously unknown fine-scale structuring in the population. These results will help direct future research efforts, conservation action and aid in the management of the Scandinavian brown bear population. LandRel thus offers an approach for detecting subtle population structure with a focus on contemporary, fine-scale analysis of continuous populations.

Keywords Continuous distribution - Non-invasive genetic sampling · INLA · Ursus arctos · Inbreeding · Conservation

\section{Introduction}

Knowledge of contemporary spatial structuring of populations is an important basis for ecological studies in addition to informing and facilitating conservation of a species (Bossart and Prowell 1998; Palsbøll 1999). With the recent advent of high-throughput technologies, studies of spatial structuring have been increasingly considering genetic structure enabling an understanding of how variations in the landscape affect gene flow. Many methods have been developed to study genetic structure and while each method is informative, there are limitations that make certain types of analysis difficult.

Most genetic structure methods are based on assignment tests where individuals are 'assigned' to a subpopulation that is most fitting to their genotype (Manel et al. 2005). In practice, most assignment methods apply a Bayesian clustering algorithm to identify population structure (Guillot and Foll 2009) such as the program Structure (Pritchard et al. 2000). Kinship-based methods are a type of assignment test that identifies and locates highly related individuals as inferred through molecular markers (Broquet et al. 2009; Palsbøll et al. 2010). Other methods include multidimensional scaling (e.g. PCA see Jombart et al. 2010) or spatial autocorrelation and are also based on allele frequencies (see Peakall et al. 2003). One common limitation of assignment methods is that they are based on the island population model (Latter 1973) and thus designed only for discrete populations. Furthermore, most methods readily identify subpopulations that are highly differentiated (i.e. with little gene flow between them), but often fail to detect structure in populations with high connectivity (Manel et al. 2005; Saenz-Agudelo et al. 2009; Lowe and Allendorf 2010). Here, we present a new method designed to detect contemporary, fine-scale spatial structure across the landscape in a continuous population, thereby offering a complementary method to the existing set of methods.

Limitations of assignment-based methods

While assignment-based methods often reveal structuring in a population, assumptions such as random sampling, Hardy-Weinberg equilibrium, and marker neutrality are common (Saenz-Agudelo et al. 2009). Violations of the assumption of marker neutrality are confounded by the variations in selective pressures on different loci, thus influencing estimations of population differentiation (Whitlock and McCauley 1999; Waples and Gaggiotti 2006). Even loci assumed to be selectively neutral can be subjected to the same selective pressures as a result of genetic linkage. Moreover, ascertainment of genetic markers, their representativeness of the whole genome, and total number of loci can have effects on resulting estimates (e.g. Helyar et al. 2010). Markers ascertained either outside the population of interest or using too few individuals to properly assess population-level allele frequencies can bias estimates of structure (i.e. ascertainment bias). Likewise, marker characteristics such as high polymorphism may mask true underlying structure (Putman and Carbone 2014). For one of the most commonly used methods, cluster-based modeling, Putman and Carbone (2014) recommend a minimum of 50 loci for reliable results. However, if the aim is to detect fine-scale structure, prohibitively more than 50 would be required (Peery et al. 2008), thus limiting studies to broad-scale structure. Furthermore, assignment methods often require a priori knowledge of source populations (Waples and Gaggiotti 2006). Finally, two confounding factors in many assignment-based methods are the presence of related individuals (Manel et al. 2005; Putman and Carbone 2014) and isolation by distance (IBD)(Blair et al. 2012; Meirmans 2012; Cushman et al. 2014; RuizGonzalez et al. 2015), which can result in family 
structure or allelic clines being confused with population structure.

Kinship-based methods

Several methods exist for estimating structure by using kinship to estimate migration rates (Palsbøll 1999; Peery et al. 2008; Saenz-Agudelo et al. 2009). Parentage methods provide accurate estimates of connectivity between two subpopulations when connectivity between subpopulations is high, which is the opposite of other assignment-based methods, making the two approaches complementary (Saenz-Agudelo et al. 2009). However, sufficient sampling coverage of candidate parents can be challenging. Several variations of kinship methods exist (see Vitalis 2002; Fontanillas et al. 2004; Broquet et al. 2009; Økland et al. 2010; Iacchei et al. 2013; Dharmarajan et al. 2014). In contrast to other assignment methods, kinship-based methods are useful in detecting finescale structuring (Saenz-Agudelo et al. 2009; Palsbøll et al. 2010). Another key advantage is that they estimate contemporary population structure based on the existing generations at the time of sampling (Palsbøll et al. 2010), whereas non-kinship assignment methods based on genetic divergence only provide estimates of structure that has accumulated over time. These estimates are, therefore, averages of processes occurring in the past tens to thousands of generations. Thus, kinship-based methods provide reliable estimates of contemporary structure in discrete populations.

The landscape relatedness (LandRel) method

Our landscape relatedness (LandRel) method is based on pairwise relatedness values interpolated over the landscape. This method is designed for detecting finescale structure in continuous and contemporary populations, but can theoretically be used for discrete populations. As LandRel relies on quantitative relatedness values, there is no distinction of kin categories (e.g. parent-offspring, full-siblings, etc.) thereby differentiating it from kinship-based methods. LandRel relies on the assumption that within a perfectly homogeneous landscape in which a population is randomly mating, the mean relatedness throughout the landscape will be uniform. Any divergence from these two assumptions will result in a heterogeneous distribution of relatedness. Consider, for example, that a fenced highway was built splitting an otherwise panmictic population in two. Individuals born near the road will no longer disperse in random directions, but will disperse alongside or away from the road on either side. This will lead to a sorting of individuals regarding relatedness. LandRel seeks to detect divergence from landscape homogeneity and random mating behaviour by testing for non-uniform distribution of relatedness throughout the landscape.

LandRel shares many of the advantages of kinshipbased methods including the ability to detect fine-scale structuring. However, unlike kinship-based methods, LandRel can be used for continuous populations and does not require a priori knowledge of source populations. Furthermore, since it is based on relatedness, there is no need to remove highly related individuals, which is necessary for some kinship-based methods (e.g. Saenz-Agudelo et al. 2009) and most other assignment methods. Additionally, kinship-based methods, particularly parentage methods, rely heavily on high quality genotyping to infer kinship. LandRel does not require knowledge of kinship making it more tolerant to genotyping errors. Furthermore, LandRel is spatially explicit thereby elucidating where in the landscape structure exists.

\section{Study system}

The Scandinavian brown bear (Ursus arctos) consists of three genetically distinct subpopulations with some connectivity between them (Manel et al. 2004; Norman et al. 2013). The two northernmost subpopulations originate from the eastern European lineage while the southernmost subpopulation is a relic of the western European Iberian lineage (Taberlet and Bouvet 1994). The population size within Scandinavia has been steadily increasing (Kindberg et al. 2011) since 1930 when a hunting-induced bottleneck reduced the size to approximately 130 individuals (Swenson et al. 1995). However, the latest population estimate revealed a decline in just a few years (Kindberg and Swenson 2014) prompting Artdatabanken (Uppsala, Sweden) to change the national status to Near Threatened. Furthermore, based on samples from up to 29 years ago, Tallmon et al. (2004) found that the southernmost population had a low effective population size and low immigration and recommended that this population be monitored for signs of inbreeding. 
This study empirically tests LandRel on the southernmost subpopulation of the Scandinavian brown bear. This population is currently estimated to consist of approximately 791 individuals (Kindberg and Swenson 2014), which has not changed significantly since the previous census (Kindberg et al. 2009), and is subjected to quota-based hunting. To our knowledge, no structuring within this subpopulation has been previously identified and we therefore assume that it is a panmictic population. However, considering that the brown bear exhibits male-biased dispersal and female philopatry (Støen et al. 2006; Zedrosser et al. 2007; Norman and Spong 2015), we expect some structure among females. Additionally, we assume the landscape to be homogeneous for the purposes of testing the method. Our expectations are thus that males will show uniform levels of relatedness throughout the landscape while females will have areas with significantly high relatedness akin to core areas.

\section{Methods}

Sample collection and genetic processing

Samples were collected in the autumn of 2012 in the neighbouring counties of Dalarna and Gävleborg in central Sweden (Fig. 1). For the purposes of conducting a census, the Swedish County Administration Board (Länstyrelsen) organised citizen volunteers to collect faeces opportunistically, place them in vials and send them with the GPS coordinates to Bioforsk (now NIBIO) (Norway) for DNA extraction. Further details of the sampling procedure can be found in Kindberg et al. (2011) and the DNA extraction protocol and individual identification in Schregel et al. (2012). DNA extracts from uniquely identified individuals were then sent to our laboratory (SLU, Umeå, Sweden).

DNA extracts from all sampled individuals were SNP (Single Nucleotide Polymorphism) genotyped at 96 loci as described in Norman and Spong (2015). The panel of SNPs included four Y-chromosome markers, three X-chromosome SNPs, four diagnostic mitochondrial markers and 85 autosomal SNPs. SNPs were ascertained de novo in brown bears across the geographic range in Sweden and included the population being analysed in this study (Norman et al.

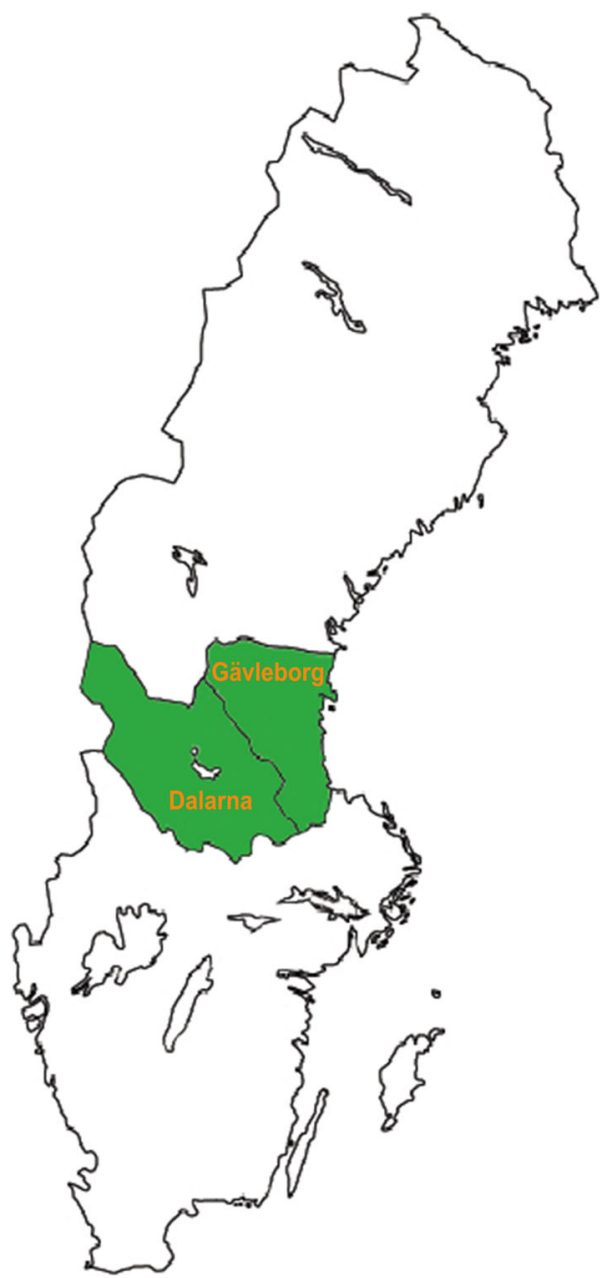

Fig. 1 Map of Sweden with the counties of Dalarna and Gävleborg, comprising the study area, highlighted in green

2013). SNPs were selected to be highly discriminatory with characteristics such as high minor allele frequency and low levels of linkage between SNPs (Norman et al. 2013).

Individuals with multiple sample locations were analysed using the median-centre of all locations to estimate the most probable home range core. Samples were collected in the autumn, when bears tend to remain within or close by their home range (Bellemain et al. 2005), thereby minimizing potential error from wandering bears. All other individuals were analysed using their single GPS point. Further details of the median-centre analysis can be found in Norman and Spong (2015). 
Population structure with PCA

We first used our genotype data to perform a principal components analysis (PCA) to discern how a commonly used and statistically rigourous method (Patterson et al. 2006) for analysing population structure performed with our data. We used the "adegenet" package version 1.4-2 (Jombart and Ahmed 2011) implemented in R (R Development Core 2013) and chose a two-dimensional analysis. Here a matrix of genotype distances was created and subsequently analysed for genetic differentiation based on eigenvalues. If there is clear population structure, points separate into distinguishable clouds. To help determine if there are true clusters, we tested the resulting values in principal component 1 to identify if it was unimodal (i.e. normally distributed), suggesting one cluster, or multimodal, suggesting two or more clusters, using a quantile-to-quantile plot (qqplot) implemented in R (R Development Core 2013).

\section{Relatedness estimates}

Estimates of relatedness between pairs of individuals were derived using the Lynch-Ritland coefficient of relatedness (Lynch and Ritland 1999) with the R package "related" version 0.8 (R Development Core 2013; Pew et al. 2014). Relatedness values (r-values) reflect the proportion of the genome that is identical by descent between two individuals. First order relatives (i.e. parent-offspring and full siblings) share approximately $50 \%$ of their genome and have an r-value around 0.50 . Second-order relatives (i.e. half-siblings and grandparent-grandoffspring) have an average r-value around 0.25 and those that are unrelated have an r-value around 0.00 . If a pair is more unrelated than expected under panmictic conditions, the r-value becomes negative. The Lynch-Ritland r-value was chosen as it has been show to outperform others in simulated and empirical studies (Thomas 2005; Csilléry et al. 2006).

\section{Interpolations}

Interpolations of relatedness across the study area were conducted using integrated nested Laplace approximations (Rue et al. 2009) with the package INLA (version 0.014) implemented in R (R Development Core 2013). INLA is an approximate Bayesian inference designed for structured latent Gaussian models; a type of additive regression model. INLA uses direct numerical integration to approximate marginal posterior densities as does the Markov Chain Monte Carlo method, but with much less computational time (Holand et al. 2013).

\section{Procedure}

Interpolations for mean relatedness were calculated based on one focal individual at a time. The geographic location of each non-focal individual was represented by the pairwise r-value with the focal individual, whereas the focal individual was not represented by any value. Interpolations were repeated in the same manner for each individual $(\mathrm{N}=412)$ so that every individual was included in an interpolation $\mathrm{N}-1$ times. The results of all interpolations were overlaid together, with the overall result being the sum of the individual-based mean relatedness values across the landscape. To determine if there existed any sexspecific patterns of relatedness, the same process was repeated using only males or only females as focal individuals. All individuals, regardless of sex, were still included as non-focal individuals.

Statistical significance was calculated using overall interpolated values at each grid point by dividing mean relatedness with root-mean-square of the standard deviation. We used an alpha level of 0.05 and, being a two-tailed test, normalised values greater than 0.975 or less than 0.025 were considered significant.

Areas of significance were in turn analysed following the same procedure as for the entire study area (global area), but only including the geographic area that contained areas of significance (local area). We use the term "global" in the context of "all-encompassing" as opposed to the global brown bear population and is used to distinguish the entire study area from the smaller "local" areas that were analysed, and that are contained within the global area. For the local areas, only individuals located within defined boundaries were included in the analysis; however, the same pairwise r-values based on the allele frequencies of the entire sample set were used. As with the global area, for each local area we first analysed all individuals and subsequently partitioned males and females. In addition to focusing on areas determined to be significant, we included an area with almost no global significance as a control. 
Fig. 2 Map of study area showing sample locations for a males $(\mathrm{n}=180)$ and b females $(\mathrm{n}=232)$ (a)

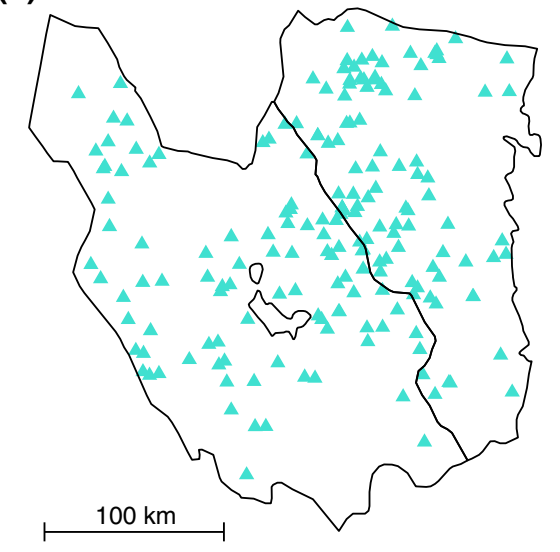

(b)

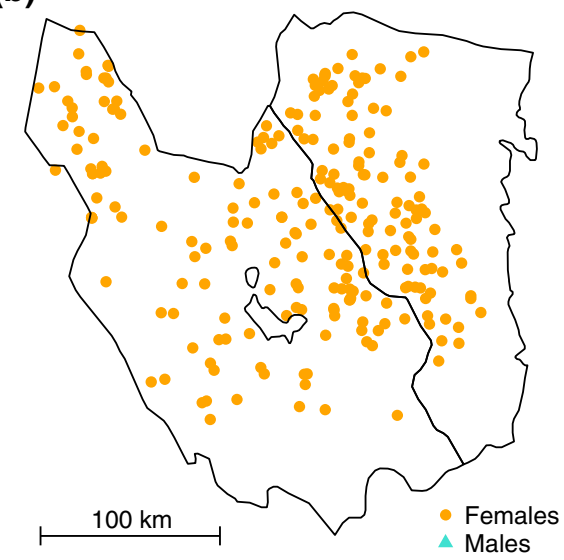

\section{INLA}

Prior to running the interpolations, relatedness coefficients were first checked to ensure a Gaussian distribution. Interpolations were performed following the guidelines of Lindgren and Rue (2015) using the spatial SPDE-model (Lindgren et al. 2011). A twodimensional mesh was created for each area being analysed. INLA was run with family set to Gaussian and using the following model:

$\mathrm{y} \sim-1+$ Intercept $+\mathrm{f}($ field, model $=$ spde $)$

where $y$ is the pairwise r-value. We ran other models with different families and including Euclidean distance as a covariate and found that the model above performed best as it resulted in the lowest deviance information criterion (DIC).

Mean relatedness and standard deviations were interpolated at every grid point on grids of $150 \times 150$ for the global area and $100 \times 100$ for the local areas. Maps were created using the R package lattice v. 0.2030 (Sarkar 2008).

\section{Inbreeding}

Areas that showed statistically significant relatedness patterns in the interpolations (i.e. the local areas) were further investigated for evidence of inbreeding. For each area, we extracted all pairwise relatedness values equal to or exceeding 0.40, to capture all first-order relatives (i.e. parent-offspring and full-siblings). We then performed a Wilcoxon rank sum test between each pairwise area to detect if there was a statistical significance between areas based on an alpha level of 0.05 .

\section{Results}

Sample collection and genotyping

We obtained DNA extracts and GPS coordinates for 412 individuals: 180 males and 232 females (Fig. 2). All individuals were genotyped successfully at all 96 SNP loci. Mean minor allele frequency of autosomal loci was 0.37 (range 0.13-0.50). The call rate excluding Y-chromosome loci was 0.997 and error rate based on heterozygous loci was $3.8 \times 10^{-4}$.

Population structure with PCA

The PCA resulted in one loose cloud with no apparent substructuring throughout all sampled individuals (Fig. 3a). The qqplot indicated a normal distribution with, perhaps, some outliers at the ends, which could represent migrants (Fig. 3b). The best interpretation of these results is thus that this group of individuals comprises one population with a few distantly related individuals and, therefore, that no substructuring exists.

\section{Relatedness estimates}

We calculated Lynch-Ritland r-values (Lynch and Ritland 1999) for each possible pairwise comparison between the 412 individuals resulting in 84,666 
(a)

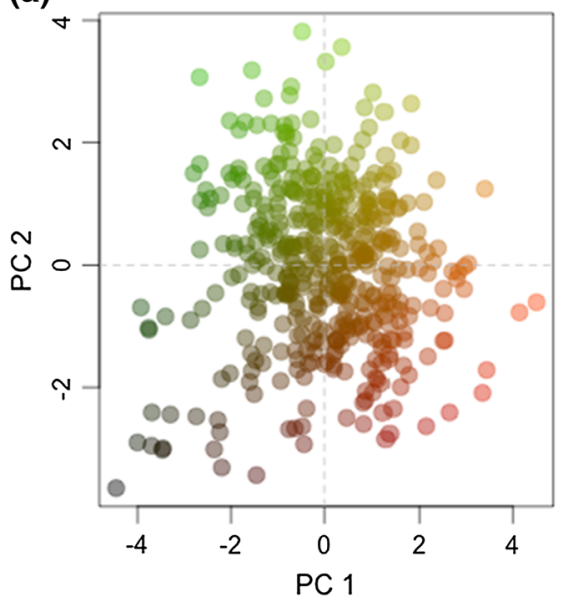

Fig. 3 Results from a principal component analysis used to identify genetic differentiation among sampled individuals. Scatterplot (a) and colours indicate genetic distance between individuals. The values from PC1 were plotted using a quantile

r-values. R-values ranged from -0.54 to 0.75 with a mean of 0.00 (SD 0.13).

\section{Interpolations}

\section{Interpretation of maps}

We generated maps showing interpolations as heat maps in which redder areas have high degrees of relatedness to all individuals in the study area ( $r-$ value $>0$ ). Green areas indicate a level of unrelatedness that would be expected in panmictic populations (r-value $\sim 0$ ). Bluer areas indicate increasing degrees of unrelatedness, which would be reflective of nonpanmictic conditions ( $r$-value $<0$ ), which could be caused either by structure in populations or isolationby-distance.

\section{Global scale (entire study area)}

Figure 4a shows the overall interpolation for the entire study area. Most of the interpolation is blue, thereby indicating that the population deviates from expectations of panmixia, which is likely attributed to effects of isolation-by-distance. Figure $4 \mathrm{~b}$ shows areas that are statistically significant (i.e. more unrelated to the population as a whole than expected by chance). The northern parts of the study area show large areas of significance. Partitioning males and females results in (b)

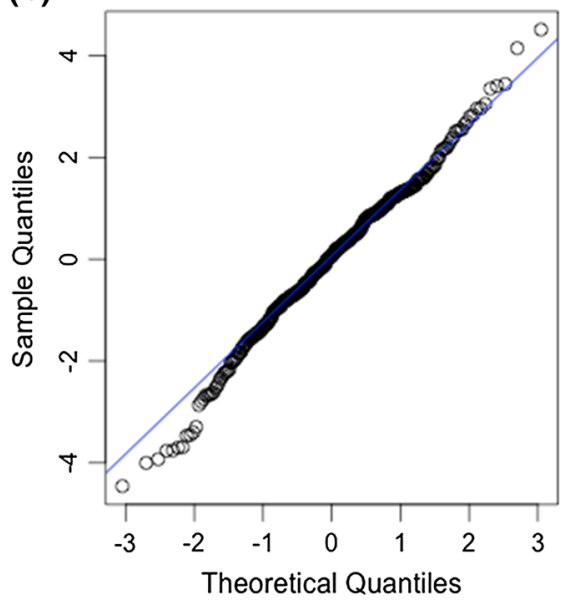

to quantile plot (b) to visually determine if they are normally distributed. The empirically derived points are the black circles and a normal distribution is expected to follow the blue line

differing patterns (see Fig. 4c-f). Males show a significant degree of unrelatedness in northern Dalarna and northern Gävleborg relative to the population as a whole, whereas females show a significantly high degree of unrelatedness in northern Gävleborg only. This suggests that individuals in these areas are segregated from the rest of the population, but it does not reveal how individuals in these areas are related to each other. To explore this, we conducted the same analysis but with a focus specifically within these areas (the local scale) (Fig. 5).

\section{Local scale (portions of the entire study area)}

Control (CA) We first analysed an area with only one small patch of significance at the global scale, which we used as a control (CA). The mean predicted relatedness across CA shows patterns of high relatedness as depicted in orange shades (Fig. 6a). This was in contrast to the results at the global scale and is likely explained by smaller geographic area and lack of isolation-by-distance. We checked for significance for each sex. Males showed no significant areas (Fig. 6b), whereas females showed large patches of significant relatedness (Fig. 6c). Since the brown bear exhibits male-biased dispersal and female philopatry, these results are consistent with our expectations: panmictic conditions for males and core areas with highly related females. 
Northern Dalarna (ND) As for CA, ND showed patterns of relatedness across the landscape (Fig. 6d). When testing for statistical significance, almost the entire area was significant. This held true for males (Fig. 6e). Females showed significance for a large proportion of the area (Fig. 6f). At the global scale, we found that males were significantly unrelated to the population as a whole (Fig. 5d). At the local scale, we found that they were significantly more related to each other than expected by chance. This indicates that the individuals in ND are segregated from the rest of the population in the study area.

Northern Gävleborg (NG) The predictions in NG follow the same pattern of relatedness across the landscape as other local areas (Fig. 6g). As for CA and unlike in $\mathrm{ND}$, there were some areas of high relatedness that were statistically significant next to areas that were not significant. However, unlike CA, the significance existed for both sexes (Fig. $6 \mathrm{~h} \mathrm{\&} \mathrm{i)}$ suggesting that males are not panmictic and hence that there is population structuring occurring.

\section{Inbreeding}

ND had significantly higher relatedness values $($ mean $=0.54)$ than both CA $($ mean $=0.50)$ and NG $($ mean $=0.51)$ areas $(\mathrm{W}=1420, \mathrm{p}$ value $=0.016$; $\mathrm{W}=2875$, $\mathrm{p}$ value $=0.0079$ respectively). The mean relatedness between $\mathrm{CA}$ and $\mathrm{NG}$ do not differ significantly $(\mathrm{W}=3009, \mathrm{p}$ value $=0.8116)$.

\section{Discussion}

We have developed a new spatially explicit method for detecting population structure in a continuous population that we call LandRel. Assuming random mating, a homogenous landscape and non-sex-biased dispersal, populations should be distributed with relatively equal levels of relatedness throughout the landscape. Where levels of relatedness are higher or lower than expected by chance, it can be inferred that one or more of the above assumptions have been violated and that population structure exists. This is what LandRel seeks to discover. Where a priori knowledge of landscape structure, dispersal characteristics or mating behaviours exist and differ from the above
Fig. 4 The first column shows interpolations of the entire study area ("global") for pairwise relatedness of a all individuals to all individuals, $\mathbf{c}$ males to all, and e females to all. The second column shows areas of statistical significance derived when the cumulated mean over the root-mean-square falls within the alpha level of 0.05 . Areas that are significant indicate that individuals in these areas are significantly more (if red) or less (if blue) related to the population as a whole than expected by chance

assumptions, adjustments of expectations of relatedness across the landscape can be made accordingly.

We empirically tested LandRel in the south-central population of the Scandinavian brown bear, which exhibits male-biased dispersal and female philopatry (Støen et al. 2006; Zedrosser et al. 2007). Our expectations were therefore that males would display equal levels of relatedness throughout the study area, while females would display areas of high relatedness, also known as core areas. Isolation-by-distance was previously tested (Mantel Test) using the same data and resulted in significance for females (p value $<0.001$ ), but not for males ( $p$ value $=0.080$ ) (see Norman and Spong 2015), thus further supporting the assumption that males are panmictic throughout the study area.

In contrast to our expectations, our LandRel results showed evidence of population structure in two areas in the northern part of the study area. This was first identified at the global scale, where individuals in these northern areas were more unrelated to the population as a whole than expected by chance. As we focused our analyses on these areas, we determined that these individuals were also significantly more related to each other than expected by chance. Since females are philopatric, and are expected to form areas of high relatedness (Støen et al. 2005), it is difficult to draw conclusions regarding population structure of females. However, in areas where males appear to be structured, females show similar patterns thereby further suggesting a barrier to gene flow, and thus population structuring for all individuals.

Since the population is continuous beyond the study area, particularly to the north and northwest, processes occurring beyond the study boundary may influence these northern areas identified as segregated. The influence may be in the form of introgression from the distinct subpopulation that exists north of the study area. Still, this does little to explain the striking differences in population-based relatedness between 

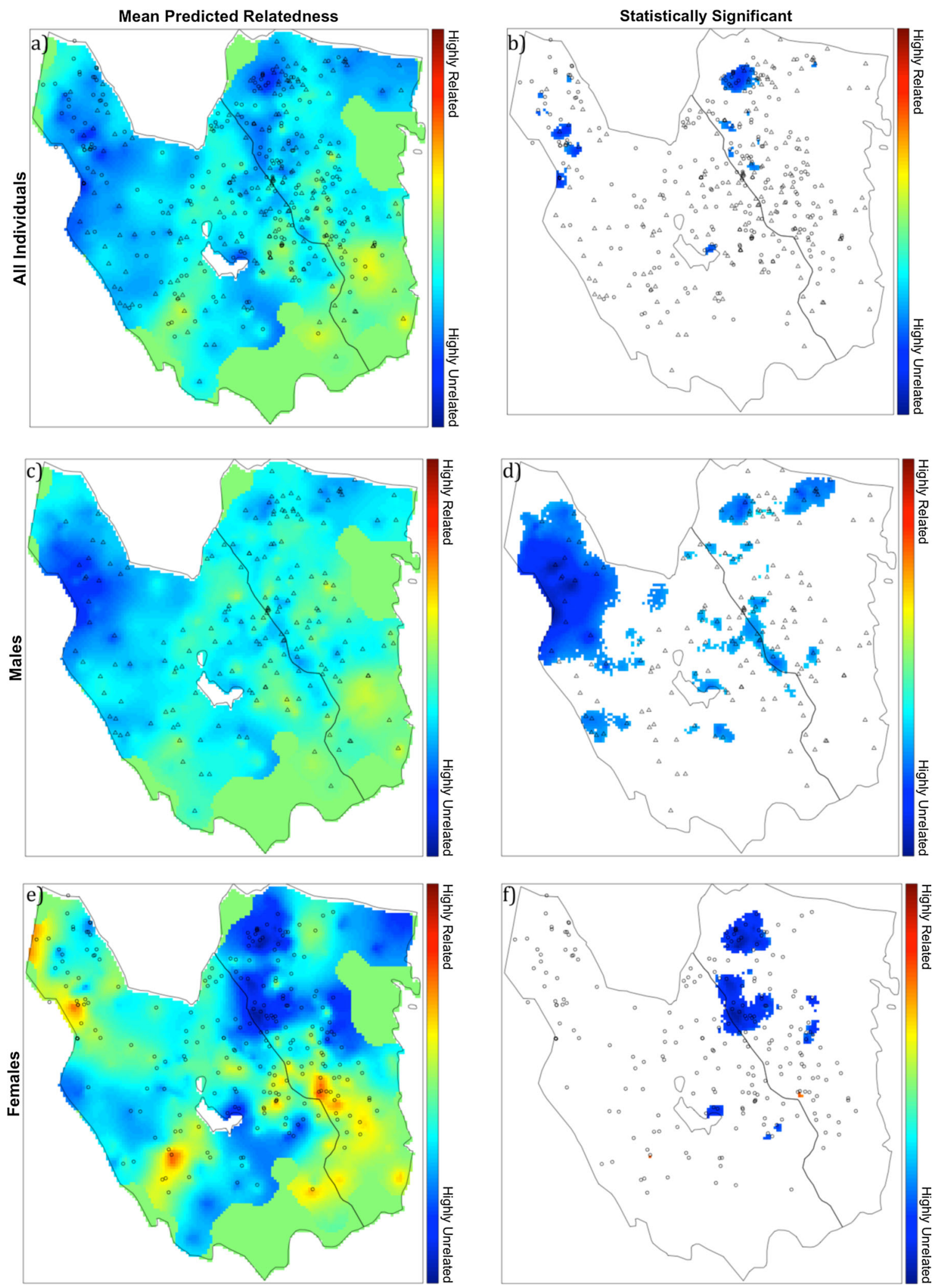


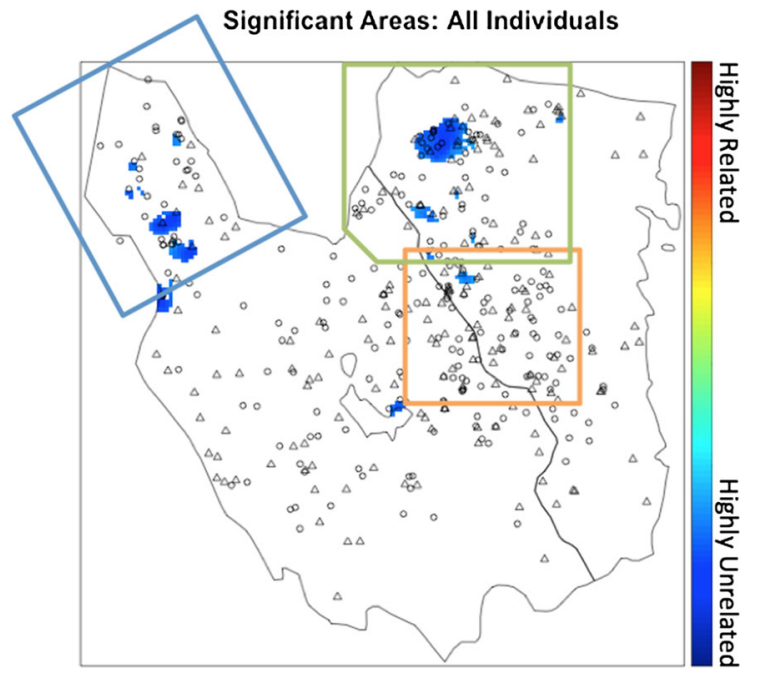

Fig. 5 Global significance map from Fig. 4 showing the three "local" areas analysed as follows: orange represents the control area (CA), blue represents northern Dalarna (ND), and green represents northern Gävleborg (NG). Males are represented as triangles and females as circles

neighbouring individuals in northern Gävleborg. Additionally, we lack information about individuals from the gap between Dalarna and Gävleborg in the north, making it difficult to determine whether the segregated individuals are a part of one continuous population, or if they are, indeed, two differentiated areas. At this time, it is unclear how biologically arbitrary sampling boundaries affect relatedness in the landscape when the population is continuous beyond the sampled boundary.

Implications for brown bear conservation and management

If the identified structure is a result of physical or behavioural isolation from the remaining population, there is a risk of inbreeding, especially if there are few individuals in the isolated area. We tested both areas that appeared to be segregated for signs of inbreeding and found that northern Dalarna had significantly higher relatedness values than the control, suggesting that inbreeding has occurred. Tallmon et al. (2004) expressed their concern for possible inbreeding in this subpopulation after detecting a low effective population size and low immigration rate. As inbreeding can have severe consequences on population fitness (Newman and Tallmon 2006), this evidence for inbreeding in northern Dalarna is of conservation concern. Though further investigation is needed to determine the cause(s) of the structuring, Nellemann et al. (2007) conducted a habitat suitability model for brown bear in an area just to the south of our local northern Dalarna area and suggested that due to the close proximity of human settlements to the west, south and east, the population may become confined. The segregated population in NW Gävleborg did not show a significant difference in relatedness values when compared with the control. However, in our analysis NW Gävleborg contains individuals that are not segregated from the main population thereby possibly balancing out the results. We therefore cannot make any conclusive statement that inbreeding has not occurred in NW Gävleborg. Our results pinpoint parts of the population that are at greater risk for reduced fitness due to isolation. Further investigation is needed to determine the cause of isolation, whether it is, for example, a barrier in the landscape or deviations from known mating or dispersal patterns.

\section{Landscape relatedness (LandRel) method}

Besides detecting population structuring, the LandRel method can be informative for other factors affecting populations. In addition to detecting areas with inbreeding, it can provide an indication of where barriers exist in the landscape irrespective of whether it is a natural barrier or a recently developed humaninduced alteration in the landscape. Additionally, if used in a monitoring programme, LandRel will be sensitive to changes within the population with no lag effect, making it an ideal monitoring tool.

One key advantage of LandRel is that it provides insights into contemporary population processes. Some of the most common methods used to analyse population structure are based on genetic differentiation between areas. This gives rise to historically derived population structure going back several to hundreds of generations. While this may be highly informative for understanding the demographic history of a species and how it has evolved, it falls short if more recent population processes need to be investigated. LandRel thus provides more immediate feedback from effects of recent anthropogenic alterations to the landscape for example.

Taking genomic data one step further from allelebased to relatedness-based analysis minimises some complications that arise with other assignment-based 

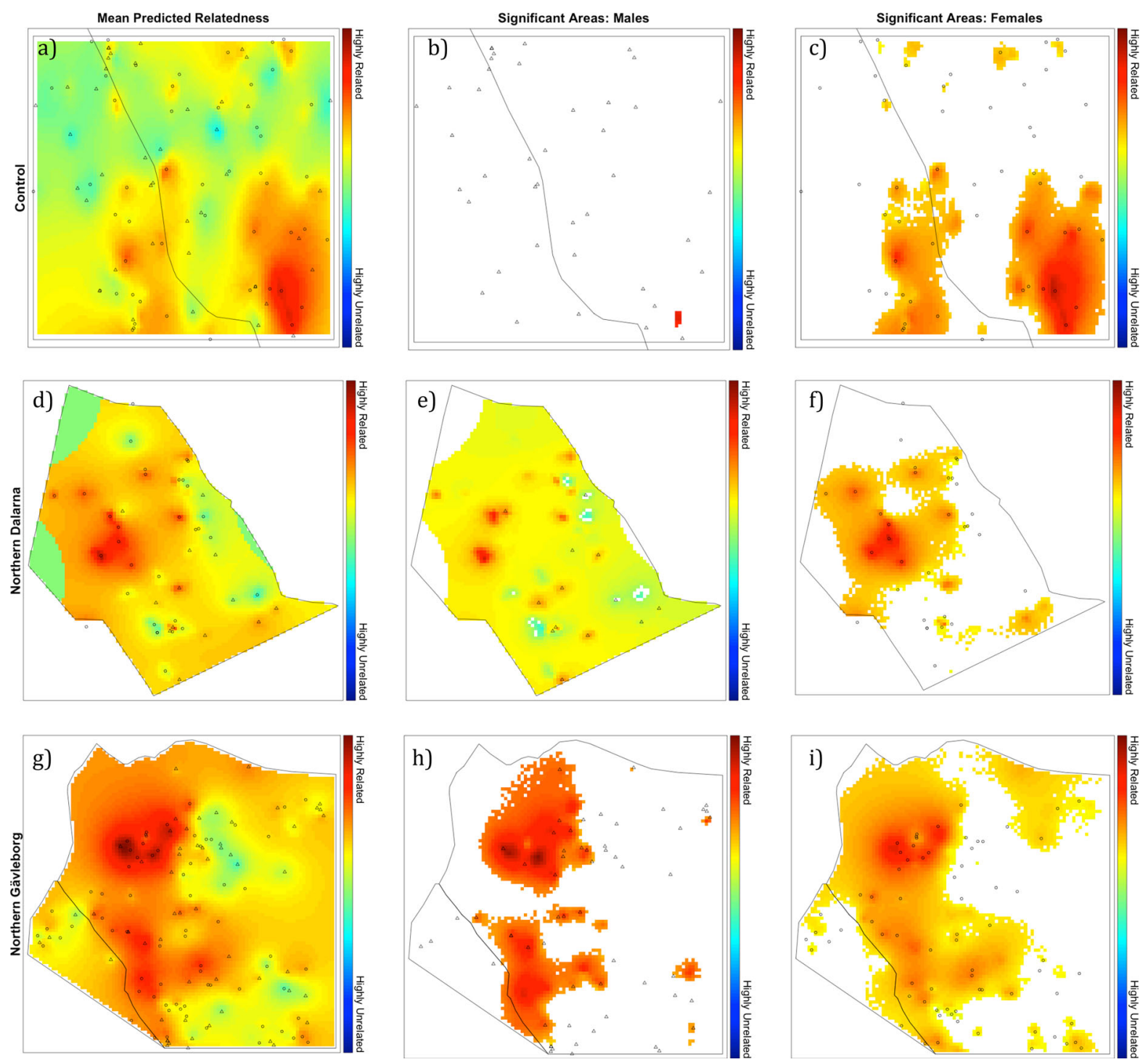

Fig. 6 The first column shows the overall interpolation for the three local areas: a control area (CA); d northern Dalarna (ND); and g northern Gävleborg (NG). The second and third column shows significant areas of relatedness for males and females respectively

methods. While the r-value itself is defined using estimates of allele frequencies in the population, the resulting comparisons between individuals should not be affected due to its relative nature. This is also true for loci that are not in Hardy-Weinberg equilibrium. Additionally, r-values are not sensitive to loci under selection. Unlike most assignment methods, LandRel will indicate if there is no structure in the population. While many kinship-based methods share these advantages, LandRel is useful for continuous populations and requires no a priori knowledge of the number of source populations unlike kinship-based methods (Iacchei et al. 2013). LandRel has much in common with the spatial autocorrelation method as developed by Peakall et al. (2003). However, LandRel uses pairwise relatedness instead of allele frequencies, thus minimizing issues associated with incorrect allele frequency estimation. In addition, LandRel results in spatially explicit structure across the landscape whereas the spatial autocorrelation method is visualized through correlograms (Peakall et al. 2003; Smouse et al. 2008). Finally, LandRel enabled us to 
detect fine-scale structuring that a commonly used method, PCA, was not able to detect.

There are some limitations with the LandRel method that should be considered. First, if there exists two panmictic subpopulations of equal size that are isolated from each other, the initial results from LandRel will not be apparent as there would appear to be an even spread of relatedness throughout. Even so, the overall interpolated mean relatedness values will end up being lower than if the entire area was panmictic due to the pairwise comparisons between individuals residing in opposite groups. However, the same result would occur if there exists isolation-bydistance. This can be investigated further by testing for isolation-by-distance and focusing the interpolation on smaller areas, as we did in this study, since any structure between areas will become more apparent. Sampling characteristics pose further limitations to LandRel, which is an issue for most landscape genetics studies (see Schwartz and McKelvey 2009). Sampling needs to be conducted at a scale large enough to encompass most natal dispersal distances in order for LandRel to be effective. For this, a priori knowledge of dispersal would be beneficial and can be accomplished using the same data needed for LandRel (see Norman and Spong 2015). While sampling intensity is not as important as scale per se, the higher the sampling intensity, the more accurate the interpolations will be. Finally, more research is needed to bring LandRel to its full potential. For example, testing how it works with various degrees of structuring and simulating different sampling intensities would identify important characteristics of the method.

Collecting samples noninvasively is one approach that enables data collection on elusive and rare species from individuals that are living without ever needing to disturb them (Taberlet et al. 1999; Waits and Paetkau 2005; Smith and Wang 2014). Since noninvasive samples collected from the environment are often degraded due to exposure to UV rays and other chemical processes, use of high quality molecular markers that are insensitive to fragmented DNA are recommended. SNPs are particularly useful for degraded DNA since they require only small DNA fragments (65-100 base pairs). Their qualitative nature also makes them less sensitive to false alleles. Additionally, it is possible to genotype using single copy detection, thereby dramatically reducing the occurrence of allelic dropouts and making it possible to accurately genotype samples with very low DNA quantities. In this study, we used SNPs with a call rate of almost 1.00 and a genotyping error rate of less than 0.0004 enabling us to maximise the amount of information extracted from the samples.

\section{Conclusion}

In this study, we show that the LandRel method can be used for identifying contemporary, fine-scale population structure within a continuous population of the southernmost Scandinavian brown bear population. It also helped identify areas with higher levels of inbreeding. As inbreeding can have negative consequences on a population, this population should be monitored regularly. LandRel is a complementary method to the many other methods used to identify population structure and has a focus on fine-scale structure across the landscape within a continuously distributed population. The primary advantage of LandRel is that it provides insight into contemporary processes within a landscape context, something that is highly sought after in ecological and conservation-oriented studies.

Acknowledgments We are grateful to ESF ConGenOmics for providing the funds for a research visit where the idea for this method was first conceived. Thanks to Torsten Nygård Kristensen and Cino Pertoldi for hosting the visit. Thanks to Håvard Rue and his group for hosting another research visit. We are grateful to NIBIO (Bioforsk) and Michael Schneider for providing us with DNA extracts and spatial data. Thanks to all the citizen volunteers and the county administration boards of Dalarna and Gävleborg for organizing the sample collection. Many thanks to Helena Königsson for her tireless work in the lab. We also thank two anonymous reviewers for their comments on an earlier version of this manuscript. This is paper \# 217 in the Scandinavian Brown Bear Research Project.

Open Access This article is distributed under the terms of the Creative Commons Attribution 4.0 International License (http:// creativecommons.org/licenses/by/4.0/), which permits unrestricted use, distribution, and reproduction in any medium, provided you give appropriate credit to the original author(s) and the source, provide a link to the Creative Commons license, and indicate if changes were made.

\section{References}

Bellemain E, Swenson JE, Tallmon D, Brunberg S, Taberlet P (2005) Estimating population size of elusive animals with DNA from hunter-collected feces: four methods for brown bears. Conserv Biol 19:150-161 
Blair C, Weigel DE, Balazik M, Keeley ATH, Walker FM, Landguth E, Cushman S, Murphy M, Waits L, Balkenhol N (2012) A simulation-based evaluation of methods for inferring linear barriers to gene flow. Mol Ecol Resour 12:822-833

Bossart JL, Prowell DP (1998) Genetic estimates of population structure and gene flow: limitations, lessons and new directions. Trends Ecol Evol 13:202-206

Broquet T, Yearsley J, Hirzel AH, Goudet J, Perrin N (2009) Inferring recent migration rates from individual genotypes. Mol Ecol 18:1048-1060

Csilléry K, Johnson T, Beraldi D, Clutton-Brock T, Coltman D, Hansson B, Spong G, Pemberton JM (2006) Performance of marker-based relatedness estimators in natural populations of outbred vertebrates. Genetics 173:2091-2101

Cushman SA, Max T, Meneses N, Evans LM, Ferrier S, Honchak B, Whitham TG, Allan GJ (2014) Landscape genetic connectivity in a riparian foundation tree is jointly driven by climatic gradients and river networks. Ecol Appl 24:1000-1014

Dharmarajan G, Beasley JC, Fike J, Rhodes OE JA (2014) Effects of landscape, demographic and behavioral factors on kin structure: testing ecological predictions in a mesopredator with high dispersal capability. Anim Conserv 17:225-234

Fontanillas P, Petit E, Perrin N (2004) Estimating sex-specific dispersal rates with autosomal markers in hierarchically structured populations. Evolution 58:886-894

Guillot G, Foll M (2009) Correcting for ascertainment bias in the inference of population structure. Bioinformatics 25:552-554

Helyar SJ, Hemmer-Hansen J, Bekkevold D, Taylor MI, Ogden R, Limborg MT, Cariani A, Maes GE, Diopere E, Carvalho GR, Nielsen EE (2010) Application of SNPs for population genetics of nonmodel organisms: new opportunities and challenges. Mol Ecol Resour 11:123-136

Holand AM, Steinsland I, Martino S, Jensen H (2013) Animal models and integrated nested Laplace approximations. G3 (Bethesda) 3:1241-1251

Iacchei M, Ben-Horin T, Selkoe KA, Bird CE, Garcia-Rodriguez FJ, Toonen RJ (2013) Combined analyses of kinship and $F$ st suggest potential drivers of chaotic genetic patchiness in high gene-flow populations. Mol Ecol 22:3476-3494

Jombart T, Ahmed I (2011) Adegenet 1.3-1: new tools for the analysis of genome-wide SNP data. Bioinformatics. doi:10.1093/bioinformatics/btr521

Jombart T, Devillard S, Balloux F (2010) Discriminant analysis of principal components: a new method for the analysis of genetically structured populations. BMC Genet 11:94

Kindberg J, Swenson JE (2014) Björnstammens storlek i Sverige 2013-länsvisa skattningar och trender. Naturvå rdsverket, Sweden. Available from: https://www.naturvards verket.se/upload/sa-mar-miljon/statistik-a-till-o/bjorn/bjor nstammens-storlek-i-sverige-2013.pdf. Accessed Nov 2015

Kindberg J, Swenson JE, Ericsson G (2009) Björnstammens storlek i Sverige 2008 - länsvisa uppskattningar och trender. Skandinaviska björnprojektet. Available from: http:// bearproject.info/publications/?limit=2\#reports. Accessed Nov 2015
Kindberg J, Swenson JE, Ericsson G, Bellemain E, Miquel C, Taberlet $\mathrm{P}$ (2011) Estimating population size and trends of the Swedish brown bear Ursus arctos population. Wildl Biol 17:114-123

Latter BDH (1973) The island model of population differentiation: a general solution. Genetics 73:147-157

Lindgren F, Rue H (2015) Bayesian spatial modelling with R-INLA. J Stat Softw 63:1-25

Lindgren F, Rue H, Lindstrom J (2011) An explicit link between Gaussian fields and Gaussian Markov random fields: the stochastic partial differential equation approach. J R Stat Soc Ser B 73:423-498

Lowe WH, Allendorf FW (2010) What can genetics tell us about population connectivity? Mol Ecol 19:3038-3051

Lynch M, Ritland K (1999) Estimation of pairwise relatedness with molecular markers. Genetics 152:1753-1766

Manel S, Bellemain E, Swenson JE, François O (2004) Assumed and inferred spatial structure of populations: the Scandinavian brown bears revisited. Mol Ecol 13:1327-1331

Manel S, Gaggiotti OE, Waples RS (2005) Assignment methods: matching biological questions with appropriate techniques. Trends Ecol Evol 20:136-142

Meirmans PG (2012) The trouble with isolation by distance. Mol Ecol 21:2839-2846

Nellemann C, Støen O-G, Kindberg J, Swenson JE, Vistnes I, Ericsson G, Katajisto J, Kaltenborn BP, Martin J, Ordiz A(2007) Terrain use by an expanding brown bear population in relation to age, recreational resorts and human settlements. Biol Conserv 138:157-165

Newman D, Tallmon DA (2006) Experimental evidence for beneficial fitness effects of gene flow in recently isolated populations. Conserv Biol 15:1054-1063

Norman AJ, Spong G (2015) Single nucleotide polymorphismbased dispersal estimates using noninvasive sampling. Ecol Evol 5:3056-3065

Norman AJ, Street NR, Spong G (2013) De novo SNP discovery in the Scandinavian brown bear (Ursus arctos). PLoS One 8:e81012

Økland JM, Haaland ØA, Skaug HJ (2010) A method for defining management units based on genetically determined close relatives. ICES J Mar Sci 67:551-558

Palsbøll PJ (1999) Genetic tagging: contemporary molecular ecology. Biol J Linn Soc 68:3-22

Palsbøll PJ, Zachariah Peery M, BéRubé M (2010) Detecting populations in the "ambiguous" zone: kinship-based estimation of population structure at low genetic divergence. Mol Ecol Resour 10:797-805

Patterson N, Price AL, Reich D (2006) Population structure and eigenanalysis. PLoS Genet 2:e190

Peakall R, Ruibal M, Lindenmayer DB (2003) Spatial autocorrelation analysis offers new insights into gene flow in the Australian bush rat, Rattus fuscipes. Evolution 57:1182-1195

Peery MZ, Beissinger SR, House RF, Berube M, Hall LA, Sellas A, Palsbøll PJ (2008) Characterizing source-sink dynamics with genetic parentage assignments. Ecology 89:27462759

Pew J, Wang J, Muir P, Frasier T (2014) related: an R package for analysing pairwise relatedness data based on codominant molecular markers. Mol Ecol Resour 15(3):557-561 
Pritchard JK, Stephens M, Donnelly P (2000) Inference of population structure using multilocus genotype data. Genetics 155:945-959

Putman AI, Carbone I (2014) Challenges in analysis and interpretation of microsatellite data for population genetic studies. Ecol Evol 4(22):4399-4428

R Development Core (2013) R: a language and environment for statistical computing. R Foundation for Statistical Computing, Vienna

Rue H, Martino S, Chopin N (2009) Approximate Bayesian inference for latent Gaussian models by using integrated nested Laplace approximations. J R Stat Soc Ser B 71:319-392

Ruiz-Gonzalez A, Cushman SA, Madeira MJ, Randi E, GomezMoliner BJ (2015) Isolation by distance, resistance and/or clusters? Lessons learned from a forest-dwelling carnivore inhabiting a heterogeneous landscape. Mol Ecol 24(20):5110-5129

Saenz-Agudelo P, Jones GP, Thorrold SR, Planes S (2009) Estimating connectivity in marine populations: an empirical evaluation of assignment tests and parentage analysis under different gene flow scenarios. Mol Ecol 18:1765-1776

Sarkar D (2008) Lattice: multivariate data visualization with R. Springer, New York

Schregel J, Kopatz A, Hagen SB, Brøseth H, Smith ME, Wikan S, Wartiainen I, Aspholm PE, Aspi J, Swenson JE, Makarova O, Polikarpova N, Schneider M, Knappskog PM, Ruokonen M, Kojola I, Tirronen KF, Danilov PI, Eiken HG (2012) Limited gene flow among brown bear populations in far Northern Europe? Genetic analysis of the east-west border population in the Pasvik Valley. Mol Ecol 21:3474-3488

Schwartz MK, McKelvey KS (2009) Why sampling scheme matters: the effect of sampling scheme on landscape genetic results. Conserv Genet 10:441-452

Smith O, Wang J (2014) When can noninvasive samples provide sufficient information in conservation genetics studies? Mol Ecol Resour 14:1011-1023

Smouse PE, Peakall R, Gonzales E (2008) A heterogeneity test for fine-scale genetic structure. Mol Ecol 17:3389-3400
Støen O-G, Bellemain E, Sæbø S, Swenson JE (2005) Kinrelated spatial structure in brown bears Ursus arctos. Behav Ecol Sociobiol 59:191-197

Støen O-G, Zedrosser A, Saebø S, Swenson JE (2006) Inversely density-dependent natal dispersal in brown bears Ursus arctos. Oecologia 148:356-364

Swenson JE, Wabakken P, Sandegren F, Bjärvall A, Franzén R, Söderberg A (1995) The near extinction and recovery of brown bears in Scandinavia in relation to the bear management policies of Norway and Sweden. Wildl Biol $1: 11-25$

Taberlet P, Bouvet J (1994) Mitochondrial DNA polymorphism, phylogeography, and conservation genetics of the brown bear Ursus arctos in Europe. Proc R Soc Biol Sci 255:195-200

Taberlet P, Waits LP, Luikart G (1999) Noninvasive genetic sampling: look before you leap. Trends Ecol Evol 14:323-327

Tallmon DA, Bellemain E, Swenson JE, Taberlet P (2004) Genetic monitoring of Scandinavian brown bear effective population size and immigration. J Wildl Manag 68:960-965

Thomas SC (2005) The estimation of genetic relationships using molecular markers and their efficiency in estimating heritability in natural populations. Philos Trans R Soc B 360:1457-1467

Vitalis R (2002) Sex-specific genetic differentiation and coalescence times: estimating sex-biased dispersal rates. Mol Ecol 11:125-138

Waits LP, Paetkau D (2005) Noninvasive genetic sampling tools for wildlife biologists: a review of applications and recommendations for accurate data collection. J Wildl Manag 69:1419-1433

Waples RS, Gaggiotti O (2006) What is a population? An empirical evaluation of some genetic methods for identifying the number of gene pools and their degree of connectivity. Mol Ecol 15:1419-1439

Whitlock MC, McCauley DE (1999) Indirect measures of gene flow and migration: FST not equal to $1 /(4 \mathrm{Nm}+1)$. Heredity (Edinb) 82(Pt 2):117-125

Zedrosser A, Stoen O, Sabo S, Swenson J (2007) Should I stay or should I go? Natal dispersal in the brown bear. Anim Behav 74:369-376 